\title{
The role of early molecular predictor in trans- plant-eligible chronic myelogenous leukemia
}

\author{
Sung-Hyun Kim
}

Division of Hematology and Oncology, Department of Internal Medicine, Dong-A University College of Medicine, Busan, Korea

Received: December 19, 2016 Accepted: December 23, 2016

\section{Correspondence to}

Sung-Hyun Kim, M.D.

Division of Hematology and Oncology, Department of Internal Medicine, Dong-A University College of Medicine, 26 Daesingongwon-ro, Seo-gu, Busan 49201, Korea

Tel: +82-51-240-2608

Fax: +82-51-246-5044

E-mail:kshmoon@dau.ac.kr

\section{See Article on Page 125-136}

Before the tyrosine kinase inhibitor (TKI) era, allogeneic stem cell transplantation (allo-SCT) was the only curative treatment for chronic myelogenous leukemia (CML). TKI discontinuation studies indicate that the use of TKIs in the treatment of CML can achieve an "operational cure," with lasting freedom from disease progression and disease-related signs and symptoms [1]. However, if the patient has developed a TKI-resistance mutation or cannot tolerate several TKIs, allo-SCT is indicated with a suitable donor. If the patient has advanced CML, allo-SCT is recommended for the blastic phase and accelerated-phase patients who have not achieved an optimal response [2].

In this issue of the Korean Journal of Internal Medicine, Lee et al. [3] report that BCR-ABL1 transcripts (MR4.5) at 3 months posttransplant predict the long-term outcomes in patients with chronic-phase CML. At 3 months posttransplant, MR ${ }^{4.5}$ was associated with significantly longer event-free survival and showed a trend to lower relapse rates. Their study enrolled 101 patients, of whom 47 were TKI-naïve at the time of transplantation (most were treated during the period before the National Health Insurance Program covered imatinib), while 51 received imatinib as their front-line therapy, and the remaining three patients received one of dasatinib, nilotinib, or bosutinib as front-line therapy. Of the patients for whom a front-line TKI failed, 17 received second-line therapy, of whom eight were treated with a third-line TKI. Although not all enrolled patients were currently indicated for allo-SCT in patients with CML, this study shows the early predictive role of MR4.5 at 3 months posttransplant. This is a meaningful finding because it suggests that early intervention with TKI therapy or modulation with immunosuppressive therapy (e.g., donor lymphocyte infusion [DLI] or withdrawal of an immunosuppressive agent) using early molecular monitoring could potentially reduce relapse after allo-SCT in transplant-eligible CML patients.

Previous studies reported that the early detection of BCR-ABL1 transcripts using polymerase chain reaction technology is associated with an increased risk of relapse $[4,5]$. However, the value of the BCR-ABL1 transcripts expressed in previous studies was not standardized. For molecular monitoring in CML, international efforts have been made to establish recommendations for the interpretation of molecular data. In 2005, experts suggested harmonizing the different methodologies 
for measuring BCR-ABL1 transcripts and using a conversion factor so that individual laboratories can express BCR-ABL1 transcript levels on an internationally agreed scale, that is, an International Scale (IS) [6]. In the work of Lee et al. [3], the value of BCR-ABLi transcripts was reported on the IS, which is another meaningful feature of this study. The problems with the detection of minimal residual disease in the posttransplant setting in hematological malignancies are the cut-off values and method of standardization for detectable molecular markers. Another problem is when we check the minimal residual disease for detecting early relapse after transplantation. After transplantation, BCR-ABLı transcripts can be detected or fluctuate at low levels in a minority of patients without obvious progression [7]. Nevertheless, this study suggests a posttransplant checkpoint and cut-off value in CML. Prospective studies must evaluate the frequency of molecular monitoring after transplantation, and validate the checkpoint and cut-off value of BCR-ABL1 transcripts. However, conducting clinical trials will be a challenge due to the lack of transplant-eligible CML patients in the TKI era.

CML is an immunologically sensitive disease following allo-SCT, as proved by the DLI effect in relapsed patients after transplant. TKI therapy after allo-SCT may affect or hinder the immune reconstitution after transplant. A recent study of a small series of patients found that nilotinib prophylaxis after allo-SCT in patients with advanced CML or Philadelphia chromosome-positive acute lymphoblastic leukemia did not jeopardize immune reconstitution or function following transplantation [8]. Further studies must evaluate the role of TKI therapy as prophylactic therapy or preemptive therapy triggered by the detection of minimal residual disease, in the posttransplantation setting in transplant-eligible CML patients.

\section{Conflict of interest}

No potential conflict of interest relevant to this article was reported.

\section{REFERENCES}

1. Hughes TP, Ross DM. Moving treatment-free remission into mainstream clinical practice in CML. Blood 2016;128:17-23.

2. Baccarani M, Deininger MW, Rosti G, et al. European LeukemiaNet recommendations for the management of chronic myeloid leukemia: 2013. Blood 2013;122:872-884.

3. Lee SE, Choi SY, Kim SH, et al. BCR-ABL1 transcripts (MR4.5) at post-transplant 3 months as an early predictor for long-term outcomes in chronic myeloid leukemia. Korean J Intern Med 2017;32:125-136.

4. Olavarria E, Kanfer E, Szydlo R, et al. Early detection of BCR-ABL transcripts by quantitative reverse transcriptase-polymerase chain reaction predicts outcome after allogeneic stem cell transplantation for chronic myeloid leukemia. Blood 2001;97:1560-1565.

5. Asnafi V, Rubio MT, Delabesse E, et al. Prediction of relapse by day 100 BCR-ABL quantification after allogeneic stem cell transplantation for chronic myeloid leukemia. Leukemia 2006;20:793-799.

6. Hughes T, Deininger M, Hochhaus A, et al. Monitoring CML patients responding to treatment with tyrosine kinase inhibitors: review and recommendations for harmonizing current methodology for detecting BCR-ABL transcripts and kinase domain mutations and for expressing results. Blood 2006;108:28-37.

7. Kaeda J, O'Shea D, Szydlo RM, et al. Serial measurement of BCR-ABL transcripts in the peripheral blood after allogeneic stem cell transplantation for chronic myeloid leukemia: an attempt to define patients who may not require further therapy. Blood 2006;107:4171-4176.

8. Varda-Bloom N, Danylesko I, Shouval R, et al. Immunological effects of nilotinib prophylaxis after allogeneic stem cell transplantation in patients with advanced chronic myeloid leukemia or Philadelphia chromosome-positive acute lymphoblastic leukemia. Oncotarget 2016 Nov 18 [Epub]. http://dx.doi.org/10.18632/oncotarget.13439. 